\title{
Effective theory approach to the measurement problem
}

\author{
William Icefield
}

(Dated: July 8, 2020 (v1))

\begin{abstract}
One understanding of the Niels Bohr's interpretation of quantum mechanics suggests that the measurement problem arises because an observer description of reality is incomplete due to lack of complete access to an entire system of observed and observer. In general, an additional and external measuring observer is required to access the entire system. Inevitable entanglement between observers, measurement apparatus and measured objects is key to such an understanding of the measurement problem. Quantum mechanics only provides a complete 'incomplete' description of reality. Nevertheless, some issues, especially providing an account of why a preferred measurement basis arises, remain. Replacing relations between observed and observer in relational quantum mechanics with those between effective (approximate) theories, one arrives at a more clear understanding of why the measurement problem arises and how a preferred measurement basis is determined. The measurement problem exists because of measurement apparatus limitations, which only allow us to formulate and verify effective theories that inevitably neglect some details of nature.
\end{abstract}

Keywords: measurement problem, interpretations of quantum mechanics, Niels Bohr, relational quantum mechanics, infinite regress, effective theory, quantum gravity 


\section{CONTENTS}

I. Introduction 2

II. Effective theory approach to measurement 5

$\begin{array}{ll}\text { A. Question of preferred basis } & 7\end{array}$

B. Born rule $\quad 8$

C. Final non-effective theory?

D. Quantization of a quantum theory 9

$\begin{array}{lr}\text { III. Conclusion } & 9\end{array}$

$\begin{array}{ll}\text { Conflict of interest } & 10\end{array}$

$\begin{array}{ll}\text { References } & 10\end{array}$

\section{INTRODUCTION}

The popular understanding of the Copenhagen interpretation, often attributed to Niels Bohr, is that an observer plays a subjective and conscious (or classical) role in a measurement process that is not captured by quantum mechanics, allowing for wavefunction collapse. Despite the common attachment of this subjectivity vision to Niels Bohr, there are doubts as to whether Niels Bohr actually advocated such a view. [1]

An alternative view of the position held by Niels Bohr is that in a measurement process, entanglement occurs between an observer, a measurement apparatus and a measured object. This technically makes these systems inseparable, but our practical limitations forbid treating these systems together. Appearance of wavefunction collapse is the consequence of this limitation.

There is infinite regress going on. Suppose subsystem $A$ is being measured. But such a measurement requires an additional measurement subsystem $M_{A}$ consisting of an observer and a measurement apparatus. But since $A$ is an incomplete picture of what is going on, a full picture requires knowing the state of $A+M_{A}$. But measuring $A+M_{A}$ requires its own measurement subsystem $M_{A+M_{A}}$. It is this infinite sequence of $A, A+M_{A}, A+M_{A}+$ $M_{A+M_{A}}, \ldots$ that makes the measurement problem difficult to resolve in this view. [2] 
Eventually, we are forced to ask the following question: instead of discussing subsystems, if we look at the entire universe or system, would it be straightforward to resolve the measurement problem? But in the above, infinite regress was suggested. Even for the entire universe, in order to know its state, one has to measure it, requiring an external measurement subsystem that would be unavailable. This may be suggested as a reason why one should not attempt to resolve the measurement problem - this is impossible as we have no access to the entire universe, and it is often said that physics should concern only about what can be measured.

But there is something peculiar about the above vision. While we may not be able to measure the entire system directly, one may measure each subsystem. While we do not know the state of the universe, this may be deduced from individual measurements, revealing full entanglement information. Therefore, in order for the Bohr vision to work, this must be made impossible or less meaningful.

It is partially this consideration that may lead one to relational quantum mechanics (RQM) [3], which suggests that the state of a subsystem is not absolute but depends on observers. In some circumstances, RQM can be considered as a natural extension of the Copenhagen interpretation in spirit of Niels Bohr. This, however, departs from how Bohr may have described the source of the measurement problem - it is rather relational aspects of quantum mechanics that generate illusions of the measurement problem in RQM. For this relativism position, there are some critical points possible, if there indeed is something absolute in reality, that can be summarized as associated with the 'determinacy problem' see $[4]$.

For RQM, collapse is simply an observer, itself being a quantum subsystem, obtaining relative information on another subsystem. Quantum mechanics requires no modification and is complete. These aspects are shared by QBism [5], though main difference exists as to treatment of objects - in RQM, all objects are on equal objective standing connected by relative information, whereas QBism focuses on subjective probabilistic experience of a single observer. Both QBism and RQM can be considered to qualify as natural extensions of the Copenhagen interpretation.

For the many-worlds interpretation - though admittedly there are many variants that we cannot fully discuss here [6-9] - collapse is simply an illusion created by only one of many worlds being accessible to us, with quantum mechanics giving us probabilistic credence one 
should assign to being in one of potential worlds. In this sense, it departs significantly from the Copenhagen interpretation. Copenhagen, RQM and many-worlds interpretations, though not surely confined to them, uphold completeness of quantum mechanics, though differing on how completeness and collapse should be interpreted.

However, there is another sub-problem to the measurement problem - the problem of preferred basis [10]. The problem of definite outcomes, outlined above, may be thought to be relatively well-addressed by most interpretations - in some sense, many interpretations agree up to empirical equivalence. The problem of preferred basis, however, is tricky. The decoherence program [11] suggests how a preferred basis may be picked, but this still is not yet a theory of preferred basis.

To use a classical probability theory analogy, state collapse may be understood as simply Bayesian updating - though with different laws of probability. There is no good classical probability analogy for preferred basis, and the quantum mechanics formalism provides no probabilistic theory of measurement basis.

In some sense, one may consider even the problem of preferred basis to be already resolved by existing interpretations. Either in RQM, many-worlds or (Neo-)Copenhagen, we may say that a quantum theory is only about predicting probability of what we may measure in a particular basis. Yet this position is difficult to square with existence of a classically determined measurement basis in actual experiments. In other words, an account of why a classically determined measurement basis emerges in quantum mechanics is missing. Even the need for such an account may be denied - in many variants of the Copenhagen interpretation, one often simply accepts the classical-quantum distinction between observer and observed.

This raises the following question: can we formulate an interpretation of quantum mechanics that provides a theory of preferred basis consistent with emergence of a classically determined measurement basis as well? We argue that the answer is yes, and this only requires a re-interpretation of relational quantum mechanics (RQM). More specifically, relations between observed and observer are replaced with relations between one effective (translated roughly as 'approximate' in ordinary language) theory at one scale to a more fundamental yet still effective theory at a different scale. It is missing details of an effective theory that generate the measurement problem, just as it is inevitable neglect of a measurement subsystem that generates the measurement problem in the 'Bohr's account' of the 
problem.

The core points of the effective theory approach to measurements are:

State collapse discontinuity in effective theory $T_{k}$ is generated

$$
\text { from continuous state evolution in another effective theory } T_{j}
$$

All effective theories are assumed to be quantum theories that require no modification to quantum mechanics, making the effective theory interpretation different from objective collapse theories $[12,13]$ that feature deviations from usual rules of quantum mechanics. However, the Penrose interpretation argument [14] that it is gravitational effects that generate the measurement problem is partially shared, if one substitutes 'missing details' with gravity.

An infinite chain of effective theories echoes infinite regress, which is why the exact source of the measurement problem cannot be traced. The infinite chain is inevitable in that practical measurement limitations allow us only to construct and verify effective theories. Just as in relational quantum mechanics, how an effective theory is to be interpreted is relative to another effective theory.

\section{EFFECTIVE THEORY APPROACH TO MEASUREMENT}

A convenient mapping can be made from concepts of relational quantum mechanics (RQM) to the effective theory approach to measurements:

$$
\begin{gathered}
S \text { relative to } M_{S} \leftrightarrow S \text { viewed from } T_{1} \\
S+M_{S} \text { relative to } M_{S+M_{S}} \leftrightarrow S+T_{1} \text { viewed from } T_{2}
\end{gathered}
$$

where $S$ refers to an observed subsystem, $M_{S}$ refers to a measurement subsystem for $S$, $M_{S+M_{S}}$ refers to a measurement subsystem for $S+M_{S}$, and $T_{i}\left(T_{1}, T_{2}, ..\right)$ refers to an

effective (translated as approximate in common language) theory. $T_{i+1}$ is considered to be a more fundamental theory than $T_{i}$, incorporating details missing from $T_{i}$. The left-hand side refers to RQM, the right-hand side refers to the effective theory approach. Furthermore, it is assumed that there is a non-invertible map from some states of $T_{i+1}$ to states of $T_{i}$ :

$$
\left|\Psi_{T_{i+1}}\right\rangle \rightarrow\left|\Psi_{T_{i}}\right\rangle
$$


Note that inevitably, only some states of $T_{i+1}$ can be mapped to states of $T_{i}$, even in approximately accurate sense.

Just as how $M_{S+M_{S}}$, before interacting with $S+M_{S}$ for a measurement, would understand $S+M_{S}$ in terms of uncollapsed wavefunction in RQM, $T_{i+1}$ would view $T_{i}$ as an incomplete deterministic theory of state evolution via the Schrödinger equation, with the state of $S$ in $T_{i}$ continuously evolving without discontinuities. The reason why $T_{i}$ experiences the measurement problem is that it misses details that $T_{i+1}$ has, just as how Bohr may have argued that the measurement problem exists because of inevitable neglect of $M_{S}$ when capturing state of $S$. Quantum effects and wavefunction collapse in $T_{i+1}$ affect effective states in $T_{i}$ via the map from states of $T_{i+1}$ to states of $T_{i}$, whenever the map can be used.

$$
\begin{aligned}
& T_{i+1} \text { thinks of itself as a probabilistic theory } \\
& T_{i+1} \text { thinks of } T_{i} \text { as an incomplete deterministic theory } \\
& \text { State collapse in } T_{i} \text { comes from new details in } T_{i+1} \\
& \text { State collapse in } T_{i+1} \text { induces state collapse in } T_{i}
\end{aligned}
$$

Note also remark (1), which is replicated:

State collapse discontinuity in effective theory $T_{k}$ is generated from continuous state evolution in another effective theory $T_{j}$

While state collapse in $T_{i+1}$ does induce state collapse in $T_{i}$, even without state collapse in $T_{i+1}$, continuous state evolution in $T_{i+1}$ can generate state collapse in $T_{i}$ right because $T_{i}$ is an incomplete effective theory.

There is an infinite chain and associated infinite regress of effective theories $T_{i}: T_{i}$ blames its measurement problem on missing details that $T_{i+1}$ has. Since there is no finite end to the chain, the definite source of the measurement problem can never be identified. For our currently known effective (quantum) field theories, the following relations can be identified:

$$
\begin{array}{r}
S \text { viewed from } T_{\text {classical }} \\
S+T_{\text {classical }} \text { viewed from } T_{1}
\end{array}
$$

where $T_{1}$ is an effective (quantum) field theory and $T_{\text {classical }}$ is a classical theory. As with the general case, $T_{1}$ would view $T_{\text {classical }}$ as an incomplete deterministic theory of state evolution. 
The only thing peculiar would be the fact that state evolution of $S$ is viewed as deterministic in $T_{\text {classical }}$ itself. Therefore, $T_{\text {classical }}$ does not seem to suffer from the measurement problem.

However, $T_{\text {classical }}$ does indeed have its version of the measurement problem. Even in the classical regime, divergences from predictions of classical physics begin to occur due to quantum effects. In this sense, it is better to think of $T_{\text {classical }}$ as a probabilistic theory as well - just that except for one outcome, all other outcomes are assigned probability of zero. Zero probability does not mean never.

Even for a classical theory, the measurement problem exists:

quantum effects do affect the classical world.

The quantization language can also be utilized. $T_{1}$ is considered as quantization of $T_{\text {classical }}, T_{2}$ as quantization of $T_{1}, T_{3}$ as quantization of $T_{2}$ and so forth: $T_{i+1}$ as quantization of $T_{i}$. In this mapping, it is made clear that $T_{i}$ is treated as a deterministic theory viewed from $T_{i+1}$, with details missing from $T_{i}$ but available in $T_{i+1}$ generating the measurement problem.

$$
T_{i+1} \text { is quantization of } T_{i}
$$

\section{A. Question of preferred basis}

The effective theory point of view makes clear of why a classically determined measurement basis emerges in quantum mechanics.

First, as with rest of the measurement problem, measurement basis for states of $T_{i}$ come from $T_{i+1}$. As aforementioned, state collapse (or its non-existence) into outcome or state $\left|\Psi_{T_{i+1}}\right\rangle$ occurs in $T_{i+1}$, and $\left|\Psi_{T_{i+1}}\right\rangle$ is translated into effective state $\left|\Psi_{T_{i}}\right\rangle$ whenever possible, which then becomes the collapse result for $T_{i}$. The natural measurement basis then surely is $\left|\Psi_{T_{i}}\right\rangle$ itself. In this sense, the problem of preferred basis is resolved.

For a usual quantum experiment, a final measurement outcome is retrieved in the classical theory regime. After translating a state in quantum theory $T_{1}$ into an effective classical state in theory $T_{\text {classical }}$, the new classical state must be in the form permissible by $T_{\text {classical }}$. The new classical state obeys evolution dictated by $T_{\text {classical }}$, except when the classical variant of the measurement problem kicks in due to quantum effects in $T_{1}$. 
The question then is why state collapse occurs in $T_{1}$ in a way as to allow the use of the map from quantum states to classical states. An explanation can only be provided by more fundamental effective theory $T_{k}$ - continuous state evolution of a combined system of a measurement subsystem and a measured subsystem in $T_{k}$ generates appearance of collapse in $T_{1}$.

As an abstraction, one may assume that a measurement apparatus, utilizing concepts of $T_{\text {classical }}$ to explore the quantum world, picks out a classically determined basis - as far as the apparatus somehow successfully carries out required interactions in $T_{k}$, one does not need to concern too much about $T_{k}$ and only focus on $T_{1}$.

\section{B. Born rule}

The question of why the Born rule has to be used to give probabilistic credence to outcomes in effective theory $T_{i}$ may be raised. Since continuous state evolution in theory $T_{k}$ can generate state collapse in theory $T_{j}$, the Born rule seems more of an epistemic principle rather than a physical rule.

In this paper, we make no attempt to give a reason why, other than the fact that the Born rule so far empirically worked well. Why the Born rule works as the best epistemic practice is explored in some other cited papers. [9, 15-17]

\section{Final non-effective theory?}

A final non-effective theory, even if it exists, cannot resolve inherent measurement apparatus limitations. For example, no measurement apparatus exists that can probe and resolve any arbitrarily high energy scale. This would be why the measurement problem cannot be completely dissolved according to the effective theory approach.

Every quantum theory is condemned to be an effective theory

due to measurement apparatus limitations. 


\section{Quantization of a quantum theory}

Given the context, quantization of a quantum theory here is more about treating a quantum theory as an incomplete deterministic theory viewed from a more fundamental yet effective quantum theory. But what exactly would be this quantization?

Multiple responses can follow - here, we only provide one possible suggestion. Given the spirit of the effective theory interpretation of the measurement problem, it is reasonable to quantize entanglement entropy. After all, in some sense, the measurement problem is about discontinuity in evolution of entanglement entropy.

The idea of entropy quantization allows us to connect to entropic gravity literature [18, 19], especially entropy extremization. [18] The general idea is that pre-quantization action $S$ of theory $T_{i+1}$ is:

$$
S=S_{\text {gravity }}+S_{\text {non-gravity }}
$$

Since one is attempting to quantize entropy, it is reasonable to think of action $S$ as prequantization entanglement entropy. Given that action $S$ is interpreted as pre-quantization entropy as well, $S_{\text {non-gravity }}$ should be about entanglement entropy in theory $T_{i}$. This is especially so, given that $T_{1}$, being one of our current empirically known effective quantum field theories, lives on Minkowski spacetime. Thus, the quantization procedure would require additionally incorporating gravitational contributions to pre-quantization entropy.

The classical least action principle then gives us how $S_{\text {gravity }}$ would need to respond to

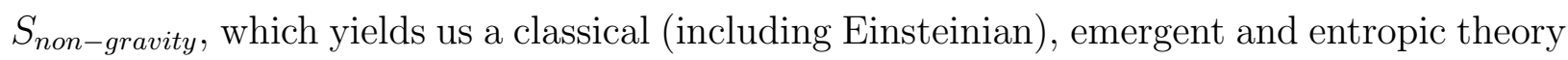
of gravity. From this point, things start to get complicated - only future works will settle these complications, and this is where we stop discussing the suggestion. For the question of whether entanglement entropy of some region can make sense in string theory, see [20].

Connections to general relativity can be made via string theory (interpreting action/prequantization entropy $S$ as string action) [21] or via entropic gravity [18, 22] in the semiclassical regime.

\section{CONCLUSION}

The effective theory approach (interpretation) to the measurement problem essentially is a re-interpretation of relational quantum mechanics - instead of relations between subsystems, 
one has relations between effective (approximate) theories on how the state of a subsystem must be interpreted.

It was postulated that every quantum theory is effective because physics is about what can be measured. Measurement apparatus limitations confine us to building and testing effective theories. And this works as a blessing in resolving the measurement problem, in that there is infinite regress - effective theory $T_{i}$ blames its measurement problem to missing details that theory $T_{i+1}$, a more fundamental yet still effective theory, has, and so forth $(i \rightarrow \infty)$.

From the point of $T_{i+1}, T_{i}$ is an incomplete deterministic theory of state evolution dictated by the Schrödinger equation. Since the measurement problem of $T_{i}$ comes from $T_{i+1}$, appearance of state collapse in $T_{i}$ can be traced to quantum effects and state collapse in $T_{i+1}$ - however, even without collapse, continuous state evolution of $T_{i+1}$ can generate state collapse in $T_{i}$.

Future works in quantum gravity would work to test the effective theory interpretation - if a theory of quantum gravity demonstrates that appearance of state collapse is generated even with continuous state evolution, then the effective theory interpretation would be confirmed.

\section{CONFLICT OF INTEREST}

The authors declare that they have no conflict of interest.

[1] Don Howard, "Who Invented the "Copenhagen Interpretation"? A Study in Mythology," Philosophy of Science 71, 669-682 (2004).

[2] Jack M. Holtzman, "A Note On Schrödinger's Cat and the Unexpected Hanging Paradox," The British Journal for the Philosophy of Science 39, 397-401 (1988).

[3] Carlo Rovelli, "Relational quantum mechanics," International Journal of Theoretical Physics 35, 1637-1678 (1996).

[4] Matthew J. Brown, "Relational Quantum Mechanics and the Determinacy Problem," The British Journal for the Philosophy of Science 60, 679-695 (2009). 
[5] Christopher A. Fuchs, N. David Mermin, and Rüdiger Schack, "An introduction to QBism with an application to the locality of quantum mechanics," American Journal of Physics 82, 749-754 (2014).

[6] Hugh Everett, "'Relative State" Formulation of Quantum Mechanics," Rev. Mod. Phys. 29, 454-462 (1957).

[7] Bryce S. DeWitt, "Quantum mechanics and reality," Physics Today 23, 30-35 (1970).

[8] Berry Groisman, Na'ama Hallakoun, and Lev Vaidman, "The measure of existence of a quantum world and the Sleeping Beauty Problem," Analysis 73, 695-706 (2013).

[9] Charles T. Sebens and Sean M. Carroll, "Self-locating Uncertainty and the Origin of Probability in Everettian Quantum Mechanics," The British Journal for the Philosophy of Science 69, 25-74 (2016).

[10] Maximilian Schlosshauer, "Decoherence, the measurement problem, and interpretations of quantum mechanics," Rev. Mod. Phys. 76, 1267-1305 (2005).

[11] Wojciech H. Zurek, "Decoherence, einselection and the existential interpretation (the rough guide)," Philosophical Transactions of the Royal Society of London. Series A: Mathematical, Physical and Engineering Sciences 356, 1793-1821 (1998).

[12] G. C. Ghirardi, A. Rimini, and T. Weber, "Unified dynamics for microscopic and macroscopic systems," Phys. Rev. D 34, 470-491 (1986).

[13] Gian Carlo Ghirardi, Philip Pearle, and Alberto Rimini, "Markov processes in Hilbert space and continuous spontaneous localization of systems of identical particles," Phys. Rev. A 42, 78-89 (1990).

[14] Roger Penrose, "On the gravitization of quantum mechanics 1: Quantum state reduction," Foundations of Physics 44, 557-575 (2014).

[15] David Deutsch, "Quantum theory of probability and decisions," Proceedings of the Royal Society of London. Series A: Mathematical, Physical and Engineering Sciences 455, 3129-3137 (1999).

[16] Wojciech Hubert Zurek, "Probabilities from entanglement, Born's rule $p_{k}=\left|\psi_{k}\right|^{2}$ from envariance," Phys. Rev. A 71, 052105 (2005).

[17] Lluís Masanes, Thomas D. Galley, and Markus P. Müller, "The measurement postulates of quantum mechanics are operationally redundant," Nature Communications 10, 1361 (2019). 
[18] T Padmanabhan, "Thermodynamical aspects of gravity: new insights," Reports on Progress in Physics 73, 046901 (2010).

[19] Erik Verlinde, "On the origin of gravity and the laws of Newton," Journal of High Energy Physics 2011 (2011), 10.1007/jhep04(2011)029.

[20] Vijay Balasubramanian and Onkar Parrikar, "Remarks on entanglement entropy in string theory," Phys. Rev. D 97, 066025 (2018).

[21] C.G. Callan, D. Friedan, E.J. Martinec, and M.J. Perry, "Strings in background fields," Nuclear Physics B 262, 593 - 609 (1985).

[22] Ted Jacobson, "Entanglement Equilibrium and the Einstein Equation," Phys. Rev. Lett. 116, 201101 (2016) 